\title{
Innovación organizativa y gestión del conocimiento en hospitales públicos en el Estado de México (2011)
}

\author{
ERENDIRA FIERRO MORENO, Ph.D. (c) $)^{1,2}$ \\ Profesora investigadora, Universidad Autónoma del Estado de México, México. \\ eren_fierro@yahoo.com \\ PATRICIA MERCADO SALGADO, Ph.D. \\ Profesora investigadora, Universidad Autónoma del Estado de México, México. \\ pat_mersal@yahoo.com
}

\section{RESUMEN}

Estudios previos constatan que la gestión del conocimiento se relaciona con la innovación, aunque es poca la atención que ha recibido la innovación organizativa, la cual es un tipo de innovación dentro de las organizaciones. El objetivo de este artículo es analizar la asociación entre innovación organizativa y gestión del conocimiento en hospitales públicos, para lo cual se aplicó un cuestionario de 55 reactivos en escala de Likert a 158 mandos medios y directivos de siete nosocomios. Se hipotetizó asociación entre ambas variables. Los resultados muestran una relación entre innovación organizativa y socialización, creación, organización y aplicación del conocimiento, en donde al menos una de éstas explica a la innovación organizativa, pero la variabilidad es exigua.

Palabras clave. Innovación organizativa; gestión del conocimiento; hospitales.

Recibido: 30-jun-11, corregido: 22-abr-12 y aceptado: 26-jun-12

Clasificación JEL: M1o

@

\footnotetext{
1 Este documento fue seleccionado en la convocatoria para enviar artículos, Call for Papers, realizada en el marco del “II Simposio Iberoamericano de Estudios Gerenciales: Una mirada interdisciplinar a la innovación”, organizado por la revista académica Estudios Gerenciales bajo la dirección de la Facultad de Ciencias Administrativas y Económicas de la Universidad Icesi; el evento tuvo lugar los días 12, 13 y 14 de octubre de 2011, en la ciudad de Cali (Colombia). Este documento fue presentado en las sesiones simultáneas del área de "Innovación en gestión del conocimiento". 2 Autor para correspondencia. Dirigir a: Facultad de Economía, Cerro de Coatepec, s/n, Ciudad Universitaria. CP. 50100, Toluca, Estado de México, México.
} 


\section{Organizational innovation and knowledge management in public hospitals in the State of Mexico (2011)}

Although previous studies have verified that knowledge management is related to innovation, little consideration has been given to organizational innovation which is one of the kinds of innovation within organizations. The purpose of this article is to analyze the relationship between organizational innovation and knowledge management in public hospitals. To this end, a 55-item questionnaire was administered to 158 middle-management officials and directors at seven different hospitals using a Likert scale. A hypothesis was formulated of the correlation between both variables. The findings show that there is a correlation between organizational innovation and the dissemination, creation, organization, and application of knowledge, acknowledging that at least one of these factors is responsible for organizational innovation, but there is scant variability.

Keywords. Organizational innovation; knowledge management; hospitals.

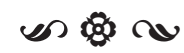

\section{Inovação organizacional e gestão do conhecimento em hospitais públicos no Estado do México (2011)}

Estudos anteriores constatam que a gestão do conhecimento está relacionada com a inovação, porém é pouca a atenção que a inovação organizacional tem recebido, sendo a mesma um tipo de inovação dentro das organizações. O objetivo deste trabalho é analisar a associação entre inovação organizacional e gestão do conhecimento em hospitais públicos, para o qual foi efetuado um questionário de 55 perguntas na escala de Likert a 158 gerentes médios e executivos de sete hospitais. Foi colocada a hipótese da associação entre as duas variáveis. Os resultados mostram uma relação entre inovação organizacional e socialização, criação, organização e aplicação do conhecimento, em que pelo menos uma dessas explica a inovação organizacional, mas a variabilidade é mínima.

Palavras-chave. Inovação organizacional; gestão de conhecimento; hospitais. 


\section{Introducción}

En los últimos tiempos la innovación y el conocimiento han constituido los máximos exponentes de la generación de riqueza y de ventajas competitivas en las organizaciones (Bradley, 1997; Pinto, Fernández, Martínez \& Kauffmann, 2006; Utterback, 1994), esto se evidencia en la importancia de los recursos organizacionales de naturaleza intangible, los cuales determinan posiciones estratégicas de crecimiento organizacional (Amit \& Schoemaker, 1993; Barney, 1991, 1992; Barney, Wright \& Ketchen, 2001; Grant, 1991; Hall, 1992; Peteraf, 1993; Ventura, 1996).

Así, la innovación se ha convertido en un esfuerzo internacional y cada vez más colectivo. México ha adoptado iniciativas cuyos logros se traducen en mejoras del diseño y la implementación de algunas políticas de ciencia, tecnología e innovación, aunque en general, los objetivos fijados no han sido alcanzados y su debilidad estructural continúa afectando el desempeño de su sistema de innovación (OCDE, 2009).

De aquí que, el sector salud mexicano requiera implementar innovaciones organizativas que son la introducción de un nuevo método organizativo en las prácticas, en la organización del lugar de trabajo o en las relaciones exteriores de la organización (OCDE \& EUROSTAT, 2005), materializadas en procesos que involucren los elementos administrativos y afecten el sistema social de la organización con la finalidad de desarrollar e implementar nuevas estructuras organizativas y procesos administrativos, así como sistemas de gestión de conocimiento (Daft, 1978; Damanpour, 1991).

Lo anterior debido a que, por un lado, no se han incrementado los recursos hospitalarios, en la misma magnitud que la demanda de servicios; y por otro, el gasto público asignado a salud es cada vez menor e insuficiente para dar respuesta a las necesidades crecientes de la sociedad. Esta situación se acentúa en los hospitales de atención a la población abierta, ${ }^{3}$ pues en tan solo cuatro años se incrementó en $310 \%$ la cobertura de atención en servicios de salud, mediante el seguro popular (INEGI, 2009).

Por ello, este artículo parte del postulado que la innovación organizativa pudiera ser sinónimo de supervivencia, estabilidad y adaptación en estas instituciones de salud ya que implica cambios significativos en las rutinas y procedimientos de gestión organizacionales, en la estructura organizacional y en la orientación estratégica de la organización (RICYT \& OEA, 2001) para reducir costos administrativos y mejorar el nivel de satisfacción en el trabajo (OCDE \& EUROSTAT, 2005).

Si bien algunos estudios han puesto de relieve el papel fundamental de la innovación tecnológica en el sector salud, la innovación organizativa parece ser una condición previa y necesaria para ello (Lam, 2005). Esto es, la innovación organizativa no constituye solamente un factor de apoyo para la innovación de producto y del servicio, sino que influye considerablemente en algunos resultados de las organizaciones tales como la mejora continua de la calidad y la eficiencia del trabajo; la reducción del tiempo de respuesta a usuarios; el incremento de la calidad de los bienes y servicios; la reducción de costos laborales e incremento de los niveles de satisfacción en el trabajo de los empleados (Community Innovation Survey, 2007); así como la apertura y respuesta al intercambio de información; y en una mayor capacidad de aprendizaje y utilización de nuevos conocimientos y tecnologías (OCDE \& EUROSTAT,

3 Población de atención abierta de acuerdo con Blanco y Maya (1997) es la población sin seguridad social; es la población no asegurada a la que se le otorgan servicios de salud. 
2005). Además, la gestión del conocimiento, al permitir la creación y la distribución de conocimiento en las organizaciones, pudiera ser un predictor de la innovación organizativa.

De aquí que el objetivo de este estudio sea analizar la asociación entre la innovación organizativa y la gestión del conocimiento en hospitales públicos en el Estado de México, los cuales deben responder a las crecientes demandas de servicio con los mismos recursos.

Con este objetivo en mente y bajo la perspectiva teórica de los recursos y capacidades (Barney, 1991; Grant, 1991; Penrose, 1959; Wernerfelt, 1984), esta investigación contribuye al conocimiento actual sobre innovación organizacional demostrando la existencia de una variable predictora de la innovación organizativa.

El resto de este documento discurre como sigue. Primero se lleva a cabo una revisión teórica sobre innovación organizativa y gestión del conocimiento así como el modelo hipotético de la asociación de ambas variables. A continuación, se establecen las hipótesis de investigación seguidas del método y los resultados estadísticos. Finalmente, se discuten los hallazgos y se presentan algunas conclusiones.

\section{Consideraciones teóricas}

1.1 Innovación organizativa en instituciones de salud

Hace más de cien años, Marshall ya incluía en sus estudios la innovación organizativa al reconocer la capacidad de la organización como ente social, para poseer, desarrollar y gestionar recursos propios; de esta manera introduce la actividad organizacional como el cuarto factor de la producción (Marshall, 1890; Sanidas, 2005).

Desde entonces se aprecia un debate teórico y práctico sobre la relevancia de la actividad organizacional con un enfoque holístico y sistémico de reestructuración que involucra y afecta el lugar de trabajo, el modelo de organización y las relaciones exteriores (Tapscott, 1997). Queda claro que las organizaciones no compiten con tendencias generales y mejores prácticas, sino mediante el desarrollo de capacidades distintivas (Nooteboom, 1999). Es en la precisión y en la forma de compartir e intercambiar conocimientos, experiencias, habilidades, información aplicada; en la generación de flujo de conocimiento adecuado y en la creación de bases de datos que registren las prácticas hospitalarias más comunes, en donde se genera innovación. Por esto la importancia de la innovación organizativa.

El término innovación está estrechamente vinculado a la investigación y al desarrollo de nuevos productos. Sin embargo, el desarrollo de tecnologías avanzadas que tienden a la innovación del producto, ya no es suficiente para ofrecer respuesta puntual a las necesidades de organizaciones específicamente de servicios.

Por lo tanto, los científicos y profesionales de la gestión han comenzado a definir la innovación en un sentido más amplio. Sugieren que las actividades de innovación pueden ser técnicas y no técnicas. Las innovaciones de productos y procesos representan las primeras; las innovaciones de servicio y/o producto y las innovaciones organizativas están dentro de las segundas (Institute Systems and Innovation Research -IIS, 2006; Kinkel, Lay \& Wengel, 2004).

Un problema importante derivado del concepto de innovación organizativa es su vaguedad; no se ha alcanzado una aceptación general de lo que es ni de sus prácticas de gestión, lo que sí se ha logrado con la innovación de producto y de proceso (OCDE \& EUROSTAT, 2005). 
Hasta ahora, la innovación organizativa es definida como la implementación de cambios en las prácticas organizacionales que mejoran la capacidad de innovación y el rendimiento de una organización (IIS, 2006). Puede lograrse a través de prácticas administrativas, procesos basados en competencias profesionales y habilidades gerenciales (Nogueira \& Marques, 2008); o bien, con la introducción de cambios en las formas de organización y gestión, así como en la incorporación de estructuras organizativas modificadas y con la implementación de nuevas orientaciones estratégicas sustancialmente cambiadas (RICYT \& OEA, 2001).

En esencia, la finalidad de la innovación organizativa es mejorar los resultados y el lugar de trabajo mediante las prácticas organizacionales, las relaciones exteriores con otras empresas o instituciones públicas, el establecimiento de nuevas formas de colaboración con organismos de investigación o clientes, la implementación de métodos de integración con los proveedores; y, la externalización o la subcontratación, por primera vez, de actividades consustanciales a la organización. Todo esto para optimizar recursos y mejorar la satisfacción en el trabajo (OCDE \& EUROSTAT, 2005).

\subsection{Sobre la gestión del conocimiento}

El conocimiento se ha convertido en el factor de producción más importante; de aquí que las organizaciones deban centrarse en su producción, adquisición, movimiento, retención y aplicación (Shaikh, 2004; Spender, 1996). Para esto, es necesario profundizar y desarrollar la gestión del conocimiento, la cual ha llegado a ser reconocida como una disciplina emergente, ${ }^{4} \mathrm{y}$ que tiene la

4 La gestión del conocimiento se encuentra en una fase embrionaria de desarrollo pero crece a un ritmo acelerado (Booker, Bontis \& Serenko, 2008); ha surgido como la estrategia que las entidades necesitan adoptar para gestionar y utilizar el conocimiento organizacional. De acuerdo con finalidad de proporcionar metodologías y guías de actuación en la práctica de administración eficaz del mismo (Benavides \& Quintana, 2003). Esto se logra mediante un conjunto de procesos que permiten utilizarlo como un factor esencial para generar valor (Tejedor \& Aguirre, 1998).

La gestión del conocimiento se refiere a la recolección de ideas, al poder de captación del conocimiento, a la conversión de conocimiento tácito en conocimiento explícito, a su codificación y transferencia (Fijalkowska, 2008), la cual debe desarrollarse intra e inter-organizativamente con la finalidad de crear valor agregado para la organización (Ordoñez, 2001).

Por lo dicho, la gestión del conocimiento se centra en facilitar y gestionar aquellas actividades relacionadas con la creación, captura, transformación y uso del conocimiento (Díaz, Contreras \& Rivero, 2009).

\section{El modelo: asociación entre innovación organizativa y gestión del conocimiento} Bajo la perspectiva teórica de recursos y capacidades, la gestión del conocimiento se considera como una capacidad interna que permite el desarrollo de la capacidad de innovación y, por lo tanto, de la innovación organizativa. En la teoría de recursos y capacidades, el crecimiento, la eficiencia, la eficacia (Daft, 1983) y, por lo tanto la ventaja competitiva de las organizaciones, deriva de los recursos (Penrose, 1959; Wernerfelt, 1984) y de las capacidades internas; esto siempre y cuando éstas sean valiosas, raras, imperfectamente imitables y no substituibles (Barney, 1991; Grant, 1991; Teece, Pisano \& Shuen, 1997), lo cual conforma una plataforma a partir de la cual la orga-

Peluffo \& Catalán (2002), la gestión del conocimiento es una disciplina emergente que se va afirmando con la aparición de nuevos paradigmas en los sistemas económicos nacionales e internacionales. 
nización puede generar nuevas capacidades (Eisenhardt \& Martin, 2000).

A partir de lo anterior, el origen, el estímulo y el incremento de la competitividad deriva, se enraíza y se define en las habilidades y factores de la organización, tales como la capacidad de innovación (Bharathi, 2007; Griffy-Brown \& Chun, 2007; Pasquini \& Mendes, 2009; Pinto et al., 2006), de la innovación organizativa y de la gestión del conocimiento, lo que contribuye a potenciar la creación de valor en la organización (Díaz et al., 2009). Esto permite nuevas formas de organización, alta tecnología, propagación de comunicación, énfasis en los recursos intangibles (Fijalkowska, 2008) $y$, en consecuencia, eficiencia y eficacia organizacionales.

Aunque el vínculo entre la investigación organizativa y la gestión del conocimiento ha sido relegado o no ha recibido suficiente atención (Fischer, 2001); algunos estudios se enfocan en la medición de esfuerzos de innovación y otros en la manera de incrementarla (McDermott \& Handsfield, 2000). $\mathrm{Al}$ respecto, se encuentra que la innovación organizativa es uno de los predictores de la gestión del conocimiento (Cheng \& Mohd, 2010).

Cabe señalar que los estudios se han centrado en la gestión del conocimiento como predictor de la innovación técnica (IIS, 2006) pero no de la innovación organizativa, lo que implicó buscar la asociación entre ambas variables a partir de dos hipótesis de investigación y con la percepción de mandos medios y directivos de hospitales públicos ubicados en el Estado de México, lo que permitió plantear las siguientes hipótesis:

Hipótesis 1 (H1). La innovación organizativa (organización en el trabajo y prácticas organizacionales) se asocia positivamente con la gestión del conocimiento (socialización, creación, organización y aplicación).

Hipótesis 2 ( $\mathrm{H2}$ ). La gestión del conocimiento tiene un efecto positivo sobre la innovación organizativa.

\section{Materiales y método}

Esta investigación fue cuantitativa con alcance exploratorio, ya que examinó la situación tal cual y, por lo tanto, su diseño fue no experimental y transversal (Creswell, 2009; Leedy \& Ellis, 2002).

En el estudio participaron siete hospitales públicos del Estado de México: cinco generales (de atención a la población abierta) y dos de alta especialidad (uno de atención a la población abierta y otro de atención a población derechohabiente). La población estudiada fueron 177 directivos y mandos medios. Se trató de una muestra no probabilística, con una tasa de participación del $88,9 \%$ de 158 individuos de tres categorías: médicos, paramédicos y administrativos; niveles jerárquicos con capacidad para conocer y definir las necesidades de innovación organizativa y gestión del conocimiento, así como para abanderar, concertar y definir el cambio planeado y dirigido para mejorar las organizaciones.

La operacionalización de la variable dependiente, innovación organizativa, se realizó con dos dimensiones: prácticas organizacionales y organización del lugar del trabajo (oCDE \& eUROSTAT, 2005). La primera se refiere a nuevos métodos para organizar las rutinas y los procedimientos de gestión de los trabajos, nuevas prácticas para mejorar el aprendizaje y la distribución del conocimiento, creación de bases de datos como prácticas de catalogación del conocimiento, políticas de formación de personal y mejora de las condiciones laborales. La organización del lugar de trabajo, estas se refieren a nuevos métodos de atribución de 
responsabilidades y de poder de decisión, división del trabajo y nuevos conceptos de estructuración e integración de actividades.

La gestión del conocimiento (variable independiente) se operacionalizó mediante cuatro dimensiones: socialización, creación, organización y aplicación del conocimiento (Chen \& Huang, 2007; Danaee \& Selseleh 2010; OCDE \& eurostat, 2005; Wensley, Cegarra-Navarro, Cepeda-Carrión \& Leal; 2011).

$\mathrm{Al}$ respecto, la socialización del conocimiento (o el conocimiento compartido) se refiere a utilizar los conocimientos actuales de la organización mediante el flujo y la dispersión del conocimiento entre las personas (Danaee \& Selseleh, 2010), con la finalidad de aumentar el valor agregado de los productos o servicios de la organización (Wensley et al., 2011). La creación del conocimiento es un proceso interminable que incluye nuevas ideas, comprensión de nuevos paradigmas y la combinación de principios aislados para el establecimiento de nuevos procesos (Danaee \& Selseleh, 2010) en donde clientes y empleados interactúan y trabajan juntos de manera interdependiente para el logro de objetivos a partir de su experiencia (Wensley et al., 2011). La organización del conocimiento se refiere al almacenamiento, registro y conservación de los conocimientos de modo que permitan a otros empleados recuperarlos como un prerrequisito para el intercambio de conocimientos (Danaee \& Selseleh, 2010). La aplicación del conocimiento es la capacidad con que los individuos, equipos y unidades en la organización logran mejoras basadas en el conocimiento (Chen \& Huang, 2007) reflejándose en los bienes o servicios de la organización (Danaee \& Selseleh, 2010).

Se aplicó un cuestionario auto-administrado integrado en dos secciones: una con las dos variables en estudio (innovación organizativa y gestión del conocimiento) y la otra con datos demográficos (sexo y edad) y organizacionales (puesto, área, antigüedad en el hospital y antigüedad en el puesto).

La medición de la variable innovación organizativa (Community Innovation Survey, 2007; OCDE \& EUROSTAT, 2005; OCDE \& Statistics Canada, 2004) se integró con 36 reactivos distribuidos en dos dimensiones: prácticas organizacionales (veinte reactivos) $(\alpha=0,94)$ y organización en el lugar de trabajo (dieciséis reactivos) $(\alpha=0,91)$. La gestión del conocimiento (Chen \& Huang, 2007; Danaee \& Selseleh, 2010; Wensley et al., 2011) se midió con diecinueve reactivos y cuatro dimensiones: socialización (siete reactivos) $(\alpha=0,69)$, creación (cuatro reactivos) $(\alpha=0,75)$, organización (cuatro reactivos) $(\alpha=0,83)$ y aplicación (cuatro reactivos) $(\alpha=0,83)$. Los reactivos se respondieron mediante una escala Likert de seis opciones que va de 1 (muy alto, totalmente de acuerdo) a 6 (muy bajo, totalmente en desacuerdo). Así, un menor puntaje se interpreta como una mejor percepción o mayor apertura a la variable.

Para ayudar a perfeccionar el cuestionario se realizó una validación de contenido a través de entrevistas con un panel de expertos, cuyas sugerencias fueron incorporadas en una segunda versión del mismo. Previo a la aplicación del instrumento con que se reportan estos resultados, se realizó una prueba piloto en noviembre de 2010 a 33 individuos de un hospital de alta especialidad de la ciudad de Toluca (México). Se obtuvo consistencia interna en cada una de las dimensiones.

Una vez aprobado el protocolo de investigación por el Departamento de Investigación en Salud del Instituto de Salud del Estado de México y por la Dirección de Educación e Investigación en Salud del Instituto de Seguridad Social del Estado 
de México y Municipios, y presentado en cada uno de los hospitales participantes, la aplicación del cuestionario se llevó a cabo de abril a julio de 2011. Se programaron sesiones grupales de cinco o más personas; se garantizó la confidencialidad y el anonimato de los respondientes. El tratamiento estadístico de los datos se realizó mediante el paquete SPSS v.17 (Statistical Package for Social Sciences).

\section{Resultados}

\subsection{Caracterización de la muestra}

El $42 \%$ de los participantes pertenecen a cinco hospitales generales y $58 \%$ a dos de alta especialidad. Los participantes provienen de diversos tamaños de hospitales (número de camas): $10 \%$ son de un hospital de dieciocho camas; $17 \%$ son de dos hospitales cada uno de treinta camas; $14 \%$ son de dos hospitales cada uno de cuarenta camas; $24 \%$ son de un hospital de noventa camas y $34 \%$ de un hospital de doscientas camas.

De la muestra, 44\% de los participantes son hombres y $51 \%$ mujeres; $40 \%$ tienen entre 36 y 45 años, $31 \%$ entre 25 y 35 años, $20 \%$ entre 46 y 55 años y $5 \%$ son personas con más de 56 años. En relación a la distribución del área, 30\% de los encuestados son del área médica, $27 \%$ al área administrativa, $23 \%$ pertenece al área médica-administrativa y $16 \%$ son paramédicos. $69 \%$ de los participantes son mandos medios y $26 \%$ son directivos.

En cuanto a la antigüedad en la institución, 53\% de los participantes son de reciente ingreso (uno a cinco años) y pertenecen al área administrativa; 23\% tienen una antigüedad de seis a diez años y son del área médica; $7 \%$ son los de mayor antigüedad (veintiséis a treinta años) y corresponden al área paramédica. $69 \%$ tiene entre uno y cinco años de antigüedad en el puesto, $20 \%$ entre seis a once años, $4 \%$ entre once a quince años; $3 \%$ entre dieciséis a veinte años; y finalmente,
$1 \%$ tiene más de veintiséis años en el puesto y pertenece al área médica.

\subsection{Comparación de medias}

Se utilizaron la Prueba Tukey y la Prueba T con la finalidad de identificar las medias significativamente distintas ( $\mathrm{sig}$. <0,05) en comparación con los datos demográficos y organizacionales. Las diferencias significativas se encuentran únicamente en la gestión del conocimiento y no en la innovación organizativa. Esto es, el sexo, la edad y la antigüedad en el hospital, sí inciden en la percepción de los participantes en relación con la gestión del conocimiento. Por el contrario, la antigüedad en el puesto, el tipo de contrato y el área no tienen impacto en la percepción de ésta.

Concretamente, los hombres (media $=3,50$; d.e. $=1,10$ ) perciben que la socialización del conocimiento (conocimiento compartido) se da en menor medida que lo que consideran las mujeres (media=3,10; d.e. $=0,77$ ). Parece que después de 56 años (media $=3,85$; d.e. $=1,10$ ) el conocimiento no fuera aplicado dentro del hospital, mientras que el personal entre 25 y 45 años (media=2,80; d.e. $=0,87)$ aplica más el conocimiento. Se considera que tanto la creación (media $=1,96$; d.e. $=0,68$ ), la organización (media $=2,40$; d.e. $=0,74)$ y la aplicación (media $=2,28$; d.e. $=0,70)$ del conocimiento es mayor en la organización cuando se tiene de 16 a 25 años de antigüedad en el hospital, pero a partir de 26 años (media=3,40; d.e. $=0,77$ ) la percepción en relación a la gestión del conocimiento decrece notablemente.

\subsection{Descriptivos (media y desviación estándar) y correlaciones Pearson \\ Como puede verse en la Tabla 1, los partici- pantes perciben que las prácticas organiza- cionales (media $=2,81$ ) y la organización en el lugar de trabajo (media=2,79) tienen un}


Tabla 1. Estadísticos descriptivos, correlaciones y coeficiente de confiabilidad

\begin{tabular}{llcccccccc}
\hline Variable & Dimension & Media & D.E. & 1 & 2 & 3 & 4 & 5 & 6 \\
\hline $\begin{array}{l}\text { Innovación } \\
\text { organizativa }\end{array}$ & $\begin{array}{l}\text { 1. Prácticas organizacionales } \\
\text { 2. Organización en el lugar de }\end{array}$ & 2,81 & 1,16 & $(0,940)$ & & & & & \\
& $\begin{array}{l}\text { trabajo } \\
\text { Gestión del } \\
\text { conocimiento }\end{array}$ & 2,79 & 1,00 & $0,869^{* *}$ & $(0,910)$ & & & & \\
& 3. Socialización (conocimiento & & & & & & & & \\
& compartido) & 3,27 & 0,95 & $0,178^{*}$ & 0,130 & $(0,690)$ & & & \\
& 4. Creación & 2,74 & 0,85 & $0,265^{* *}$ & $0,273^{* *}$ & $0,563^{* *}$ & $(0,750)$ & \\
& 5. Organización & 3,10 & 0,81 & $0,250^{* *}$ & $0,226^{* *}$ & $0,631^{* *}$ & $0,639^{* *}$ & $(0,830)$ & \\
& 6. Aplicación & 2,91 & 0,87 & $0,213^{* *}$ & $0,222^{* *}$ & $0,589^{* *}$ & $0,662^{* *}$ & $0,746^{* *}$ & $(0,830)$ \\
\hline
\end{tabular}

Nota. ${ }^{* *} \mathrm{p} \leq 0,01 ;{ }^{*} \mathrm{p} \leq 0,05$. Los índices de confiabilidad aparecen entre paréntesis en la intersección de cada variable. Fuente: Elaboración propia.

impacto considerable sobre la satisfacción en el trabajo y en la reducción de costos administrativos aunque es poco el consenso al respecto (d.e. $=1,16$ y d.e. $=1,00$; respectivamente). En cuanto a la gestión del conocimiento, se da más la creación (media $=2,74$; d.e. $=0,85$ ) y la aplicación (media $=2,91$; d.e. $=0,87$ ) que la organización (media $=3,10$; d.e. $=0,81$ ) y la socialización (media $=3,27$; d.e.=0,95), áreas donde el consenso es mayor.

Se utilizó la prueba $\mathrm{r}$ de Pearson para someter la hipótesis 1. Los patrones de correlación significativos se dieron en $87,5 \%$ de las intersecciones $(75,0 \%$ con $\mathrm{p} \leq 0,01 \mathrm{y}$ $12,5 \%$ con $\mathrm{p} \leq 0,05)$ entre ambas variables $\mathrm{y}$ van desde 0,178 hasta 0,273. La mayoría de las correlaciones entre las dimensiones de los constructos fueron de moderadas a altas $(0,563$ a 0,869$)$, lo que indica que las dimensiones con sus constructos son convergentes.

La alta correlación entre prácticas organizacionales y organización en el lugar de trabajo $(\mathrm{r}=0,869 ; \mathrm{p}<0,01)$ confirma la pertenencia de ambas dimensiones a la innovación organizativa. Aunque débil, la relevancia de las prácticas organizacionales se refleja en la correlación con las cuatro dimensiones de la gestión del conocimiento: la socialización $(\mathrm{r}=0,178 ; \mathrm{p} \leq 0,05)$, la creación $(\mathrm{r}=0,265 ; \mathrm{p} \leq 0,01)$, la organización $(\mathrm{r}=0,250 ; \mathrm{p} \leq \mathrm{0}, 01)$ y la aplicación $(\mathrm{r}=0,213$; $\mathrm{p} \leq \mathrm{0}, 01)$. Por su parte, la organización en el lugar de trabajo sólo se correlaciona significativamente con la creación $(r=0,273$; $\mathrm{p} \leq \mathrm{0}, 01)$, la organización $(\mathrm{r}=0,226 ; \mathrm{p} \leq \mathrm{0}, 01)$ y la aplicación del conocimiento $(\mathrm{r}=0,222$; $\mathrm{p} \leq \mathrm{0}, 01)$. Como también era de esperarse, en la medida en que el conocimiento se comparta o se socialice, se contribuye notoriamente a su creación $(\mathrm{r}=0,563 ; \mathrm{p} \leq 0,01)$, a su organización $(\mathrm{r}=0,631 ; \mathrm{p} \leq 0,01)$ y a su aplicación ( $\mathrm{r}=0,589 ; \mathrm{p} \leq 0,01)$. Por lo tanto, la creación de conocimiento exige su organización $(\mathrm{r}=0,639 ; \mathrm{p} \leq 0,01)$ y su consecuente aplicación $(\mathrm{r}=0,662 ; \mathrm{p} \leq 0,01)$, pero también la organización de éste facilita su aplicación $(\mathrm{r}=0,746 ; \mathrm{p} \leq \mathrm{0}, 01)$. Por lo anterior, la hipótesis 1 pudo ser soportada.

\subsection{Regresión múltiple}

Para abordar la hipótesis 2, es decir, el efecto de la gestión del conocimiento sobre la innovación organizativa verificando el efecto combinado de las variables, se realizó un análisis de regresión múltiple (ver Tabla 2).

Las cuatro dimensiones de la gestión del conocimiento (socialización, creación, organización y aplicación) contribuyen a explicar poco más del $8 \%$ de la variabilidad de las prácticas organizacionales $\left(\mathrm{R}^{2}=0,081\right)$, de la organización en el lugar de trabajo $\left(\mathrm{R}^{2}=0,084\right)$, y suponen un modelo estadís- 
Tabla 2. Regresión múltiple de la gestión del conocimiento (VI) y la innovación organizativa (VD)

\begin{tabular}{|c|c|c|}
\hline \multirow{2}{*}{ Gestión del conocimiento (vI) } & \multicolumn{2}{|c|}{ Innovación organizativa (vD) } \\
\hline & Prácticas organizacionales ${ }^{*}$ & Organización en el lugar de trabajo* \\
\hline Socialización (conocimiento compartido) & $-0,014$ & $-0,092$ \\
\hline Creación & 0,186 & 0,225 \\
\hline Organización & 0,151 & 0,104 \\
\hline Aplicación & $-0,015$ & 0,050 \\
\hline $\mathrm{R}^{2}$ & 0,081 & 0,084 \\
\hline $\mathrm{R}^{2}$ ajustada & 0,057 & 0,060 \\
\hline $\mathrm{F}$ & 3,039 & 3,489 \\
\hline Sig. & 0,011 & 0,009 \\
\hline
\end{tabular}

Nota. ${ }^{\star}$ Coeficientes estandarizados.

Fuente: Elaboración propia.

tico significativo para ambas dimensiones de la innovación organizativa (VD) $(\mathrm{F}=3,390$; $\mathrm{p}<0,01$ y $\mathrm{F}=3,489 ; \mathrm{p}<0,01)$, por lo que la hipótesis 2 pudo ser soportada.

\section{Discusión}

El objetivo de este estudio fue analizar la asociación de la innovación organizativa y la gestión del conocimiento en hospitales públicos en el Estado de México, organizaciones que deben responder a las crecientes demandas de servicio sin incremento proporcional de recursos.

Si bien la literatura sobre la gestión del conocimiento como antecedente de la innovación es cuantiosa, no así cuando se trata de la innovación organizativa. Con este estudio se detectó una asociación positiva entre las dimensiones que integran las variables en estudio, incluso de forma altamente significativa. Según la muestra de mandos medios y directivos médicos, administrativos y paramédicos participantes, la socialización, creación, organización y aplicación del conocimiento se asocian con la innovación organizativa (H1); esto es, en la medida en que la institución hospitalaria favorezca la generación de nuevas ideas y oportunidades de aprendizaje, así como la

mejora y la adquisición de conocimiento, el registro del conocimiento, el desarrollo de sistemas de información, la utilización adecuada de la experiencia y la aplicación de lo aprendido, el personal podrá recuperarlo para capitalizarlo en la generación de procesos innovadores, maneras diversas de organizar el trabajo cotidiano, estrategias para mejorar el aprendizaje y, hasta la construcción de nuevas bases de datos. Este escenario es coincidente con los resultados obtenidos por Cheng \& Mohd (2010), quienes hicieron estudios en 171 grandes empresas manufactureras de Malasia y obtuvieron resultados similares.

Es de llamar la atención que la socialización del conocimiento no refleja ningún vínculo con la organización en el lugar de trabajo, por lo que parecería que ésta no precisa de que todos compartan lo que saben, hacen y construyen en la organización.

A pesar que diversos estudios de innovación sí lo reconocen (Liao, Fei \& Liu, 2008; Nonaka, 1991; OCDE \& EURostaT, 2005; Wang \& Ahmed, 2003), este estudio identificó poca influencia (variabilidad) de la gestión del conocimiento frente a la innovación organizativa, aunque el modelo sí resultó significativo; es decir, al menos una 
de las variables de socialización, creación, organización y aplicación del conocimiento implican una relación lineal con las prácticas organizacionales y con la organización del lugar de trabajo como componentes de la innovación organizativa (H2).

\section{Conclusiones}

El significado literal de innovación es la introducción de algo nuevo. Académicos y profesionistas han dedicado esfuerzos serios para investigar que esa innovación implica mejoras sustantivas. No obstante, esas mejoras no deben ser solamente tecnológicas sino también administrativas. De esto último se ocupa la innovación organizativa.

Desde la perspectiva teórica de recursos y capacidades lo que genera valor a las organizaciones, aparte de los recursos internos, son las capacidades que introducen nuevos conocimientos o potencializan los ya existentes; por ello, la gestión del conocimiento se convierte en una capacidad interna de la organización que puede influenciar a la capacidad de innovación (Broos \& Cronjé, 2009; Liao \& Fei, 2010; Madhoushi \& Sadati, 2010; Nonaka, 1991; Wang \& Ahmed, 2003) y, por lo tanto, a la innovación organizativa.

Considerando los resultados de la investigación, lo anterior pudo ser demostrado en una muestra de mandos medios y directivos médicos, paramédicos y administrativos que laboran en hospitales públicos en el Estado de México; no obstante este estudio contribuye al conocimiento actual sobre innovación organizacional al probar la relación con la innovación organizativa.

La innovación organizativa, al ser una capacidad interna de la organización, permite la optimización de costos administrativos y la mejora en la satisfacción en el trabajo (ocde \& eurostat, 2005). De aquí que pueda decirse que esta constituye eficiencia y eficacia organizacionales, tal y como lo indica la perspectiva teórica que argumentó la asociación de las variables de esta investigación. Concretamente, si bien existe una débil asociación entre las prácticas organizacionales y la organización en el lugar de trabajo (componentes de la innovación organizativa) con la socialización, creación, organización y aplicación del conocimiento, la asociación es determinante entre las variables. Es preciso señalar que la gestión del conocimiento determina exiguamente la variabilidad de la innovación organizativa, aunque el modelo es altamente significativo, y al menos una de las variables de la gestión del conocimiento explica a la innovación organizativa.

Una posible explicación de los resultados obtenidos en este estudio es que en las instituciones hospitalarias bajo estudio, la gestión del conocimiento es parcial, o bien no es llevada a cabo de manera juiciosa o formalmente. Prueba de ello es que no hay bases de datos, ni reportes de experiencias (positivas y negativas) para evitar la repetición de errores o bien, favorecer los aciertos; o si existen, no siempre están a la disposición oportuna de los usuarios internos.

Lo anterior permite concluir que la gestión del conocimiento es un predictor de la innovación organizativa. No obstante, futuras líneas de investigación podrían estar enfocadas en llevar a cabo la evidencia empírica en otro tipo de organizaciones, con la finalidad de comprobar la asociación de las variables y poder determinar el efecto o no de la gestión del conocimiento sobre la innovación organizativa, así como en la identificación de otros predictores de ésta.

Toda vez que la innovación organizativa es dinámica, se recomiendan diseños de investigación longitudinales.

Finalmente una limitante del presente estudio fue el tamaño de muestra, lo cual recomienda considerar con precaución las 
conclusiones obtenidas de esta investigación. Una segunda limitante fue la utilización de una única fuente (instrumento de medición) para la recolección de los datos.

\section{Referencias bibliográficas}

Amit, R. \& Schoemaker, P. (1993). Strategic assets and organizational rent. Strategic Management Journal, 14(1), 33-46.

Barney, J. (1991). Firm resources and sustained competitive advantage. Journal of Management, 17(1), 99-120.

Barney, J. (1992). Integrating organizational behavior and strategy formulation research: A resource based analysis. Advances in Strategic Management, 8, 39-61.

Barney, J., Wright, M. \& Ketchen, D. (2001). The resource-based view of the firm: ten years after 1991. Journal of Management, $27(6), 625-641$.

Benavides, C. \& Quintana, C. (2003). Gestión del conocimiento y calidad total. Madrid: Diaz de Santos.

Bharathi, G. (2007). Intellectual capital statements: What do they measure and report. The ICFAI Journal of Accounting Research, 6(4), 52-64.

Blanco, J.H. \& Maya, J.M. (1997). Fundamentos de salud pública. Medellín: Corporación para investigaciones biológicas.

Booker, L., Bontis, N. \& Serenko, A. (2008). The relevance of knowledge management and intellectual capital research. Knowledge \& Process Management, 15(4), 235-46.

Bradley, K. (1997). Intellectual capital and the new wealth of nations. Business Strategy Review, 8(1), 53-62.

Broos, E. \& Cronjé, J. (2009) Information society needs of managers in a large governmental organization. Educational Technology \& Society, 12(1), 285-297.

Chen, C. \& Huang, J. (2007). How organizational climate and structure affect knowledge management-The social interaction perspective. International Journal of Information Management, 27(2), 104-118. Cheng, T. \& Mohd, A. (2010). The influence of knowledge management effectiveness on administrative innovation among Malaysian manufacturing firms. Asian Academy of Management Journal, 15(1), 63-77.

Community Innovation Survey. (2007). CIS4, Encuesta a los Estados de la Unión Europea. Recuperado el 17 de julio de 2010, de http://epp.eurostat.ec.europa. eu/portal/page/portal/product_details/ publication?p_product_code $=$ KS SF-07-116

Creswell, J. (2009). Research Design. Qualitative, Quantitative, and Mixed Methods Approaches (3a ed.). Londres: Sage.

Daft, R. (1978). A dual-core model of organizational innovation. Academy of Management Journal, 21(2), 193-210.

Daft, R. (1983). Organization theory and design. New York, NY: West.

Damanpour, F. (1991). Organizational Innovation: A Meta-analysis of effects of determinants and moderators. Academy of Management Journal, 34(3), 555-590.

Danaee, F. \& Selseleh, F. (2010). Measuring Knowledge Management Cycle: Evidence from Iran. European Journal of Scientific Research, 41(2), 297-309.

Díaz, M., Contreras, Y. \& Rivero, S. (2009). El factor humano como elemento dinamizador del proceso en la gestión de la información y conocimiento. ACIMED, 20(5), 42-55.

Eisenhardt, K.M. \& Martin, J. (200o). Dynamic capabilities: What are they? 
Strategic Management Journal, 21(10-11), 1105-1121.

Fijalkowska, J. (2008). Review of guidelines for the intellectual capital statement - how to manage and communicate the company's knowledge. Portuguese Journal of Management Studies, 13(3), 327-338.

Fischer, B. (2001). Managing Innovation: an analysis of the literature. En Academy of Management (sponsor), Spring 2001

Midwest Business Administration Association Conference, Chicago, Illinois, Estados Unidos.

Grant, R.M. (1991). The resource based theory of competitive advantage: implications for strategy formulation. California Management Review, 33(3), 114-135.

Griffy-Brown, C. \& Chun, M. (2007). Aligning business strategies and IS Resources in Japanese SMEs: A Resource-Based View. Journal of Global Information Technology Management, 10(3), 18-51.

Hall, R. (1992). The strategic analysis of intangible resources. Strategic Management Journal, 13(2), 135-144.

INEGI. (2009). Estadísticas históricas de México. Colección memoria. México. Recuperado el 2 de noviembre de 2010, de http://inegi.com.mx

Institute Systems and Innovation Research -IIs. (2006). Patterns of Organizational Change in European Industry (PORCH). Ways to Strengthen the Empirical Basis of Research and Policy. Innovation Papers, 46, 1-169.

Kinkel, S., Lay, G. \& Wengel, J. (2004). Innovation: Mehr als Forschung und Entwicklung. Wachstumschancen auf anderen Innovationspfaden. ISI (Institut Systemtechnik und Innovationsfoschung), 33, 1-12.

Lam, A. (2005). Organizational Innovation. En J. Fagerberg, D. Mowery \& R.R. Nelson
(Eds.), The Oxford Handbook of Innovation (pp. 115-147). New York, NY: Oxford University Press.

Leedy, P. \& Ellis, J. (2002). Practical Research. Planning and Design ( $7^{\mathrm{a}}$ ed.). Upper Saddle River, NJ: Merrill Prentice Hall.

Liao, S.-H. \& Fei, W.-C. (2010). System perspective of knowledge management, organizational learning, and organizational innovation. Expert Systems with Applications, 37(2), 1096-1103.

Liao, S.-H, Fei, W.-C. \& Liu, C.-T. (2008). Relations between knowledge inertia, organizational learning and organization innovation. Technovation, 28(4), 183-194. Madhoushi, M. \& Sadati, A. (2010). Knowledge Management, Antecedent of Organizational Innovation and Competitiveness. En Proceedings of the European Conference on Intellectual Capital. Disponible en http://connection.ebscohost. com/c/articles/49549037/knowledgemanagement-antecedent-organizationalinnovation-competitiveness

Marshall, A. (1890). Principles of Economics (8a ed.). Londres: Macmillan and Co. (Publicado originalmente en 1890). Recuperado el 21 de febrero de 2011, de http://www.econlib.org/library/Marshall/ marP 24.html\#Bk.Iv,Ch.X

McDermott, C. \& Handsfield R. (200o). Concurrent Development and Strategic Outsourcing: Do the rules change in Breakthrough Innovation? Journal of High Technology Management Research, 11(1), 35-57.

Nogueira, F. \& Marques, C. (2008). Organizational innovation: Research into the information/training paths of decision-makers within Hospitals. Portuguese Journal of Management Studies, 13(2), 237-354. 
Nonaka, I. (1991). The knowledge-creating company. Harvard Business Review, 69(6), 96-104.

Nonaka, I. \& Takeuchi, H. (1995). The knowledge creating company. Oxford, UK: University Press Oxford.

Nooteboom, B. (1999). Innovation, learning and industrial organization. Cambridge Journal of Economics, 23(2), 127-150.

OCDE. (2009). OCDE Reviews of Innovation Policy: Mexico. Recuperado de http:// www.oecd.org/sti/innovationinsciencetechnologyandindustry/oecdreviewsofinnovationpolicymexico.htm

ocde \& eurostat. (2005). Manual de Oslo. Guía para la recogida e interpretación de datos sobre innovación ( $3^{\mathrm{a}}$ ed.). Madrid: Grupo Tragsa.

oCDE \& Statistics Canada. (2004). Measuring Knowledge Management in the business sector: First steps. Paris: OCDE Publishing. Ordoñez, P. (2001). La gestión del conocimiento como base para el logro de una ventaja competitiva sostenible: la organización occidental versus japonesa. Investigaciones Europeas de Dirección y economía de la Empresa, 7(3), 91-108.

Pasquini, M. \& Mendes, M. (2009). Organizational Values and Innovative Organizational. Knowledge Creation. RAC, 13(1), 36-56.

Peluffo, M. \& Catalán, E. (2002). Gestión del Conocimiento y su aplicación al Sector Público. Serie Manuales CEPAL/ILPES (Instituto Latinoamericano y del Caribe de Planificación Económica y Social), 22, 1-92.

Penrose, E. (1959). The Theory of the growth of the firm. Oxford, uk: Blackwell.

Peteraf, M. (1993). The cornerstone of competitive advantage: A resource based view. Strategic Management Journal, 14(3), 179-191.
Pinto, J., Fernández, R., Martínez, L. \& Kauffmann, G. (2006). Análisis del énfasis en la innovación en la implantación del "Middle-Up-Down Management Model": Un estudio evolutivo en las empresas manufactureras del país vasco. Aspectos metodológicos y empíricos. Estudios Gerenciales, 22(101), 37-59. Disponible en http://www.icesi.edu.co/revistas/ index.php/estudios_gerenciales/article/ view/207

RICYT \& OEA. (2001). Manual de Bogotá: Normalización de indicadores de innovación Tecnológica en América Latina y el Caribe. Bogotá: Autores. Recuperado el 14 de agosto de 2010, de http://www. uis.unesco.org/Library/Documents/ Bogota\%2oManual_Spa.pdf

Sanidas, E. (2005). Organizational Innovations and economic growth. Londres: Edward Elgar Cheltenham.

Shaikh, J. (2004). Measuring and reporting of intellectual. Capital performance analysis. The Journal of American Academy of Business, 4(1-2) 439-448.

Spender, J.C. (1996). Making knowledge the basis of a dynamic theory of the firm. Strategic Management Journal, 17 (Winter Special Issue), 45-62.

Tapscott, D. (1997). La Economía Digital: las nuevas oportunidades y peligros en un mundo empresarial y personal interconectado en red. Bogotá: McGraw-Hill.

Teece, D.J., Pisano, G. \& Shuen, A. (1997). Dynamic capabilities and strategic management. Strategic Management Journal, 18(7), 509-533.

Tejedor, B. \& Aguirre, A. (1998). Proyecto Logos: investigación relativa a la capacidad de aprender de las empresas españolas. Boletín de Estudios Económicos, 53(164), 231-249. 
Utterback, J.M. (1994). Mastering the $d y$ namics of innovation. Boston, MA: Harvard Business School Press.

Ventura, V. (1996). Análisis dinámico de la estrategia empresarial: Un ensayo interdisciplinario. Oviedo, España: Servicio de Publicaciones de la Universidad de Oviedo.

Wang, C.L. \& Ahmed, P.K. (2003). Organizational Learning: a critical review. The Learning Organization, 10(1), 8-17.

Wensley, A., Cegarra-Navarro, J., CepedaCarrión, G. \& Leal, A. (2011). How entrepreneurial actions transform customer capital through time: Exploring and exploiting knowledge in an openmindedness context. International Journal of Manpower, 32(1), 132-150.

Wernerfelt, B. (1984). A resource-based view of the firm. Strategic Management Journal, 5(2), 171-180. 